\title{
VON MARSYAS BIS KÜBLBÖCK. EINE KLEINE GESCHICHTE UND THEORIE MUSIKALISCHER WETTKÄMPFE
}

\author{
Dietrich Helms
}

\begin{abstract}
»Während er schreit, wird die Haut ihm über die Glieder gerissen, Und er ist nur eine Wunde: es rinnt ihm das Blut von dem Körper, Bloß sind die Sehnen, enthüllt, die Adern vibrieren ihm ohne

Jegliche Haut; man könnte die Eingeweide, die zucken, Zählen und auch in der Brust die deutlich schimmernden Fasern"
\end{abstract} (Ovid, Metamorphosen, Buch 6, V. 387-391).

Der Wettstreit als Horrortrip. Da findet jemand ein Instrument, übt darauf wie besessen, ein Autodidakt zweifellos, ein Typ von ganz unten, der sich erheben will über seine Verhältnisse, seine eigene Bedeutungslosigkeit. Schließlich fordert er den besten Musiker heraus, den er kennt - einen Gott der Musik. Doch er hat keine Chance, denn die Jurorinnen stehen auf der Seite der Macht. Sie selber sind Teil des herrschenden Systems, singen zur Musik des Herausgeforderten. Und so muss der Verlierer für seine Anmaßung bezahlen. Er wird wahrhaft bloßgestellt, das Innerste nach außen gekehrt, schonungslos dem fremden Blick offenbart. Er wird geschunden, seine Haut zu Markte getragen - und dort vergeht sie, weht im Wind und zuckt noch im Reflex, wenn bestimmte Melodien ertönen - an einem Baum auf dem Markt von Kelainai in Phrygien.

Die antike Sage vom Wettstreit des Silens Marsyas gegen den Gott der Harmonie Apollon, mit der meine Geschichte des musikalischen Wettkampfs in der europäischen Kultur beginnt, ist heute nach Deutschland sucht den Superstar noch genauso aktuell, wie sie es zu Ovids Zeiten war. Musik eignet sich zum Wettbewerb, doch sie braucht ihn nicht. Es gibt lange Abschnitte in der Musikgeschichte und im Verlauf der Geschichte viele musikalische Kulturen, für die Wettkämpfe ohne Bedeutung sind. Natürlich lässt sich behaupten, Konkurrenz unter Musizierenden habe es immer gegeben. Dass uns 
die Musikgeschichte jedoch als eine Folge von Vergleichen und Ranglisten erscheint, liegt auch daran, dass die Feststellung, dass jemand der Beste gewesen sei, charakteristisch für unsere Art der Musikhistoriographie ist, die im Kern immer noch als Heroengeschichte geschrieben wird. Sind nicht die ersten bekannten Komponisten der abendländischen Musikgeschichte der »optimus organista « Leonin und der »optimus discantor « Perotin? Und wird nicht das erste »Ausnahme-Genie (Finscher 2003: Sp. 1214) der Musikgeschichte, Josquin des Prez, vor allem durch einen Brief des Girolamo da Sestola legitimiert, in dem dieser schreibt, er komponiere besser als Isaac allerdings nur dann, wenn er wolle?

Im musikologischen Anekdotenschatz wird über beinahe jeden, dem die Geschichte den Titel eines großen Meisters zuschreibt, von mindestens einem virtuosen Scharmützel berichtet. Auch wenn ihr Wahrheitsgehalt gelegentlich nur anhand zweifelhafter Quellen zu belegen ist, sind diese Geschichten offenbar unentbehrlich für den Nachweis der Qualitäten, die den Meister oder gar das Genie auszeichnen. Legendär ist der musikalische Vergleich zwischen Georg Friedrich Händel und Domenico Scarlatti im Jahre 1707, von dem John Mainwaring allerdings erst 1760 berichtet (1987: 66-67; vgl. auch Pont 1991). ${ }^{1}$ Nicht ganz vertrauenswürdig auch der dennoch in allen Bach-Biographien wiedergegebene Bericht vom Wettstreit zwischen Johann Sebastian Bach und Louis Marchand, dem sich der französische Virtuose durch Flucht entzogen haben soll (Geck 2000: 14). Gesicherter sind die Auseinandersetzungen Mozarts² - so z.B. im Jahr 1781 mit Muzio Clementi (vgl. Komlós 1989) -, wenn auch Mozarts Briefe alles andere als ausgewogene Quellen sind. Die Aufzählung ließe sich beliebig fortführen z.B. mit Haydns Sieg über seinen Schüler Ignaz Joseph Pleyel 1792 oder Beethovens Beweis seines Genies gegen Daniel Gottlieb Steibelt 1800.

Die »Sieger « dieser Konkurrenzen wurden per Akklamation durch die Anwesenden festgestellt und nicht offiziell durch eine Jury proklamiert. So gab es auch keine erklärten Verlierer. Von Preisgeldern ist selten die Rede;

1 Scarlatti musste selbstverständlich verlieren, zumal Mainwaring zuvor schon davon berichtet, Scarlatti (ob Domenico oder Alessandro geht aus der Quelle nicht hervor) habe bereits bei einem ersten Treffen in Venedig festgestellt, dass nur zwei so auf dem Flügel spielten: der berühmte "Sachse« und der Teufel (Mainwaring 1987: 62).

2 Weitgehend legendär bleibt dagegen die wohl bekannteste Konkurrenz Mozarts mit Antonio Salieri. Der historisch gesicherte Kern der Legende ist allerdings die Aufführung von Mozarts Einakter Der Schauspieldirektor in der Folge von Salieris Prima la musica e poi le parole 1786, die allein durch die zeitliche Nähe den Vergleich zwischen den Komponisten herausforderte. Weitere Vergleiche Mozarts sind u.a. mit Sixt Bachmann auf der Orgel (1766) und Johann Wilhelm Häßler (Orgel und Klavier) überliefert (s. Siegele 1999 und Keym 2002: Sp. 845). 
es ging vermutlich vor allem um Ehre und Ansehen, die sich allerdings durchaus auszahlen konnten. Die Vergleiche dienten Publikum und Musikern in höfischen Kammern und großbürgerlichen Salons vor allem als Gegenstand von Gesprächen (vgl. das Beispiel in Komlós 1989: 7), wobei - und hier wird ihr eigentlicher sozialer Sinn gelegen haben - mit den jeweiligen Geschmacksurteilen und der Kunst, diese zu begründen, Konkurrenzen innerhalb des Publikums ausgetragen werden konnten, die halfen einen Konsens zu bilden. ${ }^{3}$

Hier geht es jedoch nicht um die Beschreibung eines Phänomens, dass ich zur Unterscheidung Konkurrenz nennen möchte, und dass tatsächlich ein universales Prinzip der Kommunikation ist. Als solches sagt es jedoch kaum etwas über die Spezifik musikalischer Kommunikationssysteme aus. Huizingas (1956: 158) Behauptung, kein kultureller Bereich sei stärker vom Prinzip des Wettbewerbs durchzogen als die Musik, ist nur aufrecht zu erhalten, wenn man, wie er, Konkurrenzen, Parteienstreite (wie den Buffonistenstreit, die Auseinandersetzungen zwischen Gluckisten und Piccinnisten oder die Fehden zwischen Wagnerianern und Brahminen) und tatsächliche Wettbewerbe undifferenziert nebeneinander stellt. In der Tat haben alle diese Auseinandersetzungen eines gemeinsam: Sie definieren Grenzen sozialer Kommunikationssysteme. Da sie jedoch hierzu unterschiedliche Mechanismen verwenden und unterschiedlich in sozialen Systemen wirken, sind sie kaum unter einen Begriff zu subsumieren. An dieser Stelle soll es ausschließlich um die Untersuchung von Wettbewerben gehen, die ich als stark formalisierte und regularisierte Vergleiche definiere, die eine im Vorhinein bestimmte, institutionalisierte Entscheidungsinstanz und einen festgelegten Preis für den Gewinner aufweisen.

3 Der direkte Vergleich war vor der Erfindung der Schallaufzeichnung die einzige Möglichkeit des Publikums, instrumentale Fähigkeiten und schließlich auch individuelle Interpretation differenzieren zu lernen, Geschmack auszubilden und zu demonstrieren. Die im 18. Jahrhundert zunehmenden Berichte von Konkurrenzen sind eine Begleiterscheinung der fortschreitenden Trennung der Systeme von Musikern und Hörern, die mit der Etablierung des bürgerlichen Konzerts abgeschlossen war. Im Salon waren diese Grenzen weniger starr. Musiker im Konzert konnten sich allein durch ihre in der Interpretation ausgedrückte Individualität differenzieren. Ihre Hörer wiederum mussten lernen, diese Individualität zu erkennen und gleichzeitig Geschmack an Interpretationen als neues soziales Distinktionsmittel zu entwickeln. Konkurrenzen wie die beschriebenen dienten der diskursiven Annäherung an das, was guter Geschmack war resp. genannt wurde. Die Musiker erfuhren aus erster Hand, was ihre Hörer von ihnen erwarteten - eine Rückkoppelung, die im bürgerlichen Konzert kaum noch möglich ist. Um 1800 übernahm das Musikfeuilleton diese Funktion. 


\section{Theorie: Einige Vorbemerkungen}

Ich möchte die These aufstellen, dass musikalische Wettbewerbe immer dann für soziale Systeme oder gar die gesamte Gesellschaft wichtig werden, wenn Kommunikation durch oder mit Musik gefährdet ist. Die systemtheoretische Kommunikationstheorie Niklas Luhmanns, von der mein Modell angeregt ist, geht von der aus dem Konstruktivismus übernommenen Annahme aus, dass das Zustandekommen von Kommunikation im Prinzip unwahrscheinlich ist. Da versuchen zwei Bewusstseine miteinander in Kontakt zu treten, sich gegenseitig zu beeinflussen, obwohl beide füreinander völlig undurchschaubar sind. Man schaut dem anderen eben nur vor den Kopf, man kann seine Reaktionen beobachten, nicht jedoch, was er wirklich denkt. Die Evolution hat allerdings, so Luhmann, Mittel geschaffen, diese Unwahrscheinlichkeit von Kommunikation wahrscheinlicher zu machen: Medien (Luhmann 1984: 220). Diese Definition von Medien ist ungewohnt, jedoch für die Beschreibung von Kommunikationsprozessen durchaus fruchtbar, weil sie gegensätzliche Phänomene des Alltagsbegriffs »Medium« (z.B. das »Medium Sprache« als Mittel der Kommunikation und das »Medium Radio« als Kanal der Kommunikation) in einer Funktion zusammenführt. Luhmann geht von drei zentralen Problemen der Kommunikation aus: Verbreitung, Verstehen und Erfolg. Jedes wird durch ein spezifisches Medium gelöst.

Das Problem der Verbreitung entsteht aus der Unwahrscheinlichkeit, dass eine Mitteilung den anderen über Orte und Zeiten hinweg überhaupt erreicht. Dieses Problem wird durch Verbreitungsmedien wie die Schrift oder auch die Schallaufzeichnung gelöst.

Das Problem des Verstehens entsteht durch die Unwahrscheinlichkeit, dass zwei Kommunizierende den Eindruck haben, tatsächlich dasselbe zu meinen. Sie haben keine Möglichkeit, die Gedanken des anderen zu lesen. Ihr gegenseitiges Verstehen können sie nur feststellen, indem sie die Reaktionen ihres Gegenübers auf ihre Mitteilung beobachten. Entsprechen diese Reaktionen den eigenen Erwartungen, müssen sie von Verstehen ausgehen. Medien wie die Sprache machen Verstehen wahrscheinlich, da durch einen komplexen Code die möglichen Reaktionen des Gegenübers festgelegt und stark begrenzt werden. Medien, die Verstehen herstellen, werden in der Systemtheorie Luhmanns Verstehensmedien genannt.

Das dritte Problem ist der Erfolg einer Kommunikation, d.h. die Unwahrscheinlichkeit, dass ein Gegenüber in der Kommunikation in genau der Weise auf eine Mitteilung reagiert, wie man es wünscht. Dieses Problem wird durch Erfolgsmedien gelöst, wie z.B. die Macht, das Geld oder die Liebe. Sie 
fließen als symbolische Generalisierungen (z.B. durch Gesten der Macht, Versprechen finanzieller Belohnung oder Zeichen von Liebe) in die Kommunikation mit ein und können den anderen motivieren, das Gewünschte zu tun.

Niklas Luhmann hat die Evolution der Kommunikation als ein »hydraulisches Geschehen der Repression und Verteilung von Problemdruck « beschrieben (Luhmann 1984: 219). Damit beschreibt er die Beobachtung, dass mit einem Problem der Kommunikation, das von einem sozialen System gelöst wurde, ein anderes umso drängender wird. Durch die Entwicklung komplexer Codes z.B. wird das Verstehen wahrscheinlicher, die möglichen Anschlusshandlungen eindeutiger und in der Anzahl reduziert. Gleichzeitig verhindert jedoch eben diese Komplexität eine weite Verbreitung - man denke nur an wissenschaftliche Fachsprachen. So können komplexe Codes auch dazu genutzt werden, die Zahl der Personen, die das System tragen, klein zu halten und gleichzeitig das (Ein-)Verständnis unter diesen zu fördern.

Benutzt ein System dagegen ein Verbreitungsmedium wie den Notendruck, um das Problem der Reichweite von Kommunikation zu lösen, wird gleichzeitig das Verstehen erschwert, die Zahl möglicher Anschlusshandlungen wächst. Die Notation machte es Komponisten möglich, über räumliche und zeitliche Distanzen hinweg mit Musikern zu kommunizieren. Gleichzeitig gaben sie damit die Möglichkeit aus der Hand, die Reaktionen der Musiker auf ihre Mitteilung in Noten, das Verstehen, zu beobachten, zu kontrollieren und gegebenenfalls auch zu korrigieren. Ohne die Erfindung der Notenschrift gäbe es keine Diskussion um Interpretationen, keine Musikkritik und keine Musikwissenschaft im heutigen Sinne.

Gelingt es einem System schließlich, das Problem des Erfolgs zu lösen, werden wiederum das Verstehen und auch die Verbreitung unwahrscheinlicher. Ein komponierender König wird nie Gewissheit haben, aus welchem Grund seine Höflinge seine Kompositionen loben: Ist es ihre musikalische Qualität oder die Macht, die er symbolisiert? Trägt letzteres tatsächlich entscheidend zum Erfolg bei, bleibt die Verbreitung wahrscheinlich auf den Machtbereich des königlichen Komponisten beschränkt. Ein ähnliches Beispiel lässt sich für das Erfolgsmedium Liebe konstruieren: Die Mitglieder einer Boygroup können nie wissen, ob ihr Erfolg auf eine bestimmte Eigenart ihrer Songs oder auf ihr gutes Aussehen, ihre (ver-)liebenswürdige Show zurückgeht. Ist es tatsächlich auch und vor allem die Liebe, grenzen sie damit die Verbreitung ihrer Songs auf all diejenigen ein, die auf das Liebesangebot ansprechen. 
Man kann die These Luhmanns vom »hydraulische[n] Geschehen der Repression und Verteilung von Problemdruck « anschaulich als ein System kommunizierender Röhren beschreiben, das aus der Physik bekannt ist. Wird der Flüssigkeitsspiegel in einer der untereinander verbundenen Röhren gesenkt, muss er unweigerlich in den anderen beiden ansteigen. ${ }^{4}$

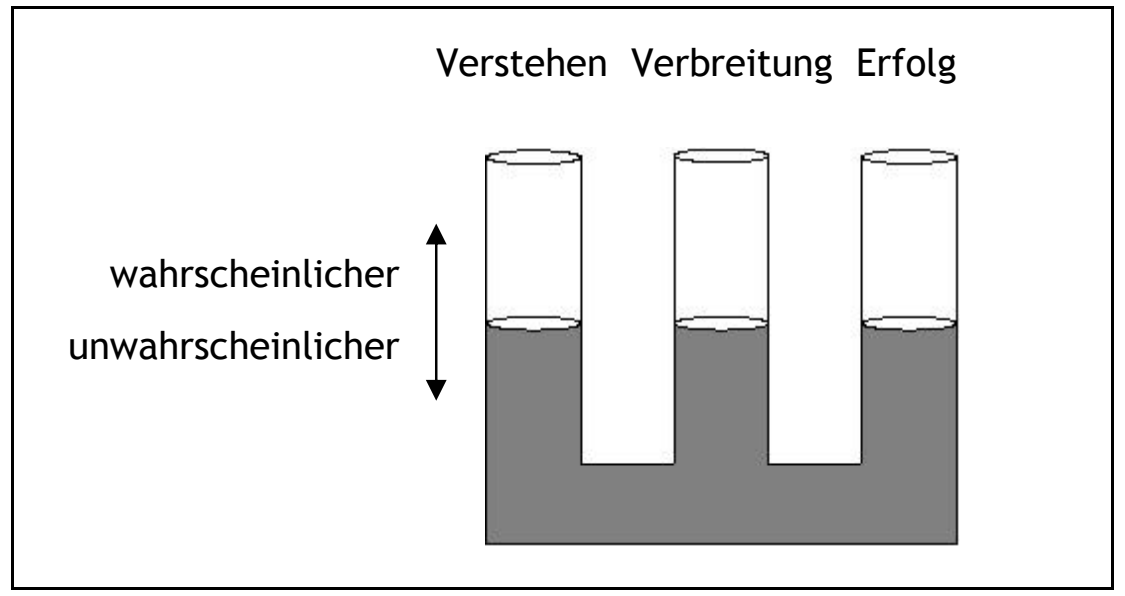

Abbildung 1: Modell des »hydraulischen Geschehens « der Repression und Verteilung von Problemdruck in der Kommunikation

Auf dieser theoretischen Grundlage lässt sich eine These für die Beschreibung der Funktion musikalischer Wettbewerbe formulieren:

Wettbewerbe helfen sozialen Systemen immer dann, ein Gleichgewicht wieder herzustellen, wenn in der Kommunikation die Verbreitung so vereinfacht und so überaus wahrscheinlich wird, dass die Eindeutigkeit des Verstehens durch die anwachsende Kontingenz gefährdet ist. Sie tun dies, indem sie die individuellen Codes des Siegers mit dem Erfolgsmedium (Definitions-)Macht ausstatten.

Eindeutigkeit herrscht vor allem in einfachen Kommunikationssystemen, wenn z.B. Musiker und Hörer eine Einheit bilden (wie etwa in Reigentänzen), wenn Kommunikationssysteme überschaubar und Codes eindeutig sind. Ein Gegeneinander mit und durch Musik wird erst dann wahrscheinlicher, wenn Kommunikation komplexer wird, wenn sich Teilsysteme ausdifferenzieren und gegeneinander abschließen: Komponisten von Musikern, Musiker von Hörern, Hörer von anderen Hörern.

4 Ob man dieses Bild so weit treiben kann, dass man hieraus rechnerische Vorhersagen auf die Zunahme des Problemdrucks in den anderen Röhren ableiten kann (z.B. in der Art, dass sich bei drei gleich starken Röhren die verdrängte Flüssigkeit, die den Problemdruck repräsentiert, zu jeweils 50 Prozent auf die beiden anderen Röhren verteilt), sei dahingestellt. 
Eine Gefahr für das Verstehen, d.h. für die Eindeutigkeit möglicher Anschlüsse, besteht immer dann, wenn durch die Verbreitung Möglichkeiten struktureller Koppelung zwischen Systemen geringer werden, wenn die Chancen einer gegenseitigen Verstehenskontrolle der Kommunikationspartner abnehmen und die verwendeten Codes dadurch immer beliebiger werden. In einem Wettbewerb einigt sich das System auf »gute« und »schlechte « bzw. wahrscheinlichere und unwahrscheinlichere Codes, gibt ersteren Eindeutigkeit und schließt letztere entweder aus dem System aus oder gibt ihnen nachrangige, weniger wahrscheinliche Bedeutungen. Es wird mit dem Sieger ein symbolischer Konsens hergestellt, der mit genügend Macht ausgestattet ist, zukünftige Kommunikationen - Kompositionen, Interpretationen oder auch Publikumsreaktionen - in seinem Sinne zu determinieren.

Ich werde meine These an vier Stationen der Musikgeschichte erläutern, die gleichzeitig wichtige Daten einer Geschichte des Wettbewerbs in der Musik darstellen: den Agonen des antiken Griechenlands, den Puis der französischen Trouvères und den damit vergleichbaren Singschulen der Meistersinger, den Nachwuchs- und Amateurwettbewerben seit dem Beginn des 19. Jahrhunderts und schließlich den aktuellen Castingshows.

\section{Selbstdefinition: Griechische Agon(i)e}

Organisierte Wettbewerbe begegnen uns in der griechischen Antike bereits dort, wo Mythen und Legenden in Geschichten und dann in Geschichte übergehen. Bereits die ältesten schriftlichen Quellen, in die Jahrhunderte alte mündliche Überlieferung einfloss, berichten wie selbstverständlich von musischen Wettbewerben. Homer erzählt im 8. Jahrhundert v. Chr. in der Odyssee, wie Alkinoos, König des mythologischen Volkes der Phaiaken, gymnische Wettkämpfe zu Ehren des Odysseus ausrichtete. Nachdem der Held alle Disziplinen außer dem Laufen gewonnen hat, gibt Alkinoos zu, dass sein Volk zwar keine guten Boxer und Ringer hervorgebracht habe, im Laufen, Tanzen und Singen jedoch alle anderen übertreffe. Dies möge Odysseus seinen Freunden berichten, wenn er wieder zuhause sei (Homer, Odyssee VIII, 246-67).

Glaubt man den autobiographischen Aussagen in den Texten Hesiods (um 700 v. Chr.), war der Dichter, der sich selbst als einen Bauern beschrieb, den die Musen das Anfertigen von Hymnen lehrten, der erste bekannte Sieger eines musischen Wettkampfs, der weder Gott noch mythologische Gestalt war. Er erwähnt in seinen Erga (650-662), dass er bei den Spielen 
zur Beisetzung des Königs von Chalkis, Amphidamas, mit einem Hymnos den Preis gewann.

Seit dem 7. Jahrhundert sind Wettkämpfe für Kitharoden bei den alle acht Jahre stattfindenden Pythischen Spielen in Delphi und bei dem Fest des Apollon Karneios in Sparta belegt (West 1992: 19). Der erste Superstar dieser Zeit war der Kitharode Terpander. Er gewann die ersten Spiele in Sparta im Gesang zur Kithara zur Zeit der 26. Olympiade (676-672 v. Chr.), wie Athenaios von Naukratis (Deipnosophistae, 635e) um 200 n. Chr. aus einem Text des Dichters Hellanicos über die Sieger der Karneia zitiert. Mit Siegen bei vier aufeinander folgenden Wettkämpfen prägte Terpander die Pythien für eine ganze Generation. Pseudo-Plutarch beschreibt noch in römischer Zeit seinen Ruhm als Gründervater des Gesangs zur Kithara (Ps.-Plutarch, Peri mousices, 1132E).

Im frühen 6. Jahrhundert wurden die großen panhellenischen Feste durch weitere musische Wettkämpfe aufgewertet. Es gab neben den zahlreichen Disziplinen für Dichter und Rezitatoren, d.h. z.B. für Rhapsoden, Tragöden und Komöden, viele Wettbewerbe für Kitharisten und Kitharoden, Auleten und Auloden, d.h. für Instrumentalsolisten und Sänger zu Instrumenten, für Chorgesang und Instrumentalisten, die Chöre begleiteten. Bei den Panathenaeen in Athen wurden vermutlich seit 525 v. Chr. Rhapsodenwettbewerbe organisiert, bei denen in einer Art rezitatorischem Staffellauf die vollständige Illias und die Odyssee vorgetragen wurden (West 1992: 19). Von den vier großen panhellenischen Festen, den Olympien, den Pythien, den Isthmien und den Nemeen, blieben nur die Olympischen Spiele die gesamte Zeit ihres Bestehens in der Antike ausschließlich gymnische Wettkämpfe (Herz 1990: 175). ${ }^{5}$

Neben den großen, den Göttern gewidmeten Festen von panhellenischer Strahlkraft, deren Sieger prestigeträchtige, symbolische Preise sowie eine ganzen Reihe von rechtlichen Privilegien gewannen, entstanden auf lokaler Ebene eine Vielzahl kleinerer Spiele, für die Geldpreise ausgelobt wurden. Sie fanden im Gegensatz zu den nur alle vier bis acht Jahre veranstalteten panhellenischen Spielen meist jährlich statt oder wurden auch nur einmalig zu besonderen Anlässen wie z.B. Trauerfeiern veranstaltet. Die große Zahl von Agonen, die finanzielle Entlohnung versprachen, begünstigte die Entwicklung eines Berufsmusikertums, das sich in mächtigen Verbänden so genannter Techniten organisierte, die wiederum eigene Agone organisierten.

5 Das änderte sich allerdings in den neuzeitlichen Olympischen Spielen mit den Spielen von Stockholm 1912, bei denen zum ersten Mal ein Kunstwettbewerb ausgeschrieben wurde (Heinze 2005: 34). Vgl. auch den Beitrag von Fred Ritzel in diesem Band. 
Die Techniten hatten einen ähnlichen Status und ähnliche Rechte wie Priester (Kübler 1934). Agone waren immer zuerst kultische Handlungen, Gottesdienste, Annäherungen an Gottheiten, die sich ihre Göttlichkeit durch ständige Konkurrenz erkämpfen und erhalten mussten. Die Bewohner des Olymps lebten es denen vor, die an sie glaubten: Ihre Macht wurde ständig herausgefordert, musste immer wieder im Kampf bestätigt werden. Der unbedeutende Quellgott Marsyas zweifelte die Herrschaft Apollons an und musste dafür bitter bezahlen. Die Musen, im Wettstreit zwischen Marsyas und Apollon zwar nicht die Unparteiischen, aber doch die Jurorinnen, fühlten sich ihrerseits durch die Kunst des Kitharisten Thamyris herausgefordert. Sie blendeten ihn und verwirrten seinen Geist, so dass er für immer verstummte (Homer, Illias II, 594-600, oder Ps.-Plutarch, Peri mousices, 1132B). Auch Orpheus stellte eine Provokation göttlicher Macht dar, die nicht ungesühnt blieb. ${ }^{6}$

Die Mythen machen deutlich: Wer das Sagen hat, will auch das Singen haben - und umgekehrt. Macht kann auch als Definitionsmacht bei der Festlegung musikalischer Codes bewiesen werden. Die Musenkünste waren ein Attribut der Göttlichkeit. Die Funktion der musiké wird auch noch von Pseudo-Plutarch als Kommunikation mit den Göttern angesehen (Peri mousices, 1131D-E). Nicht nur die Pythagoreer verehrten die Musik als Abbild der göttlichen Harmonie, die sie in der ganzen Schöpfung erkannten. Platon stellt im 4. Jahrhundert v. Chr. dar, dass ein Dichter oder Musiker nicht aus sich selbst heraus schöpft, sondern von den Musen besessen wird. Aus seinem Mund spricht die Gottheit (Platon, Ion, 533b-535a). Platon verwendet in seiner Beschreibung ein aufschlussreiches Bild: Die göttliche Inspiration funktioniere wie ein Magnetstein, der Eisenringe anzieht und sie magnetisiert, so dass diese wiederum andere Eisenringe anziehen. So inspirierten die Musen den Dichter mit göttlichem Feuer, dieser seine Interpreten und diese wiederum die Hörer. Die Beliebtheit eines Stücks und die Kunstfertigkeit seiner Ausführung werden so zu Zeichen göttlicher Inspiration.

Man kann diese Begeisterung für Wettkämpfe einem »allgemeinen Gefühl des Wettbewerbs «, welches das »gesamte Leben Griechenlands [...] von Anfang an « durchdrang (Herz 1990: 175), ${ }^{7}$ zuschreiben und die Erklärung hierfür in religiösen Vorstellungen suchen. Doch verkürzt eine solche Erklärung die Ursachen, die sich hinter einer so ausgeprägten Kultur des Wett-

6 In römischer Zeit berichtet Ovid vom Wettkampf der Musen gegen die Periden. Die Nymphen als Jurorinnen sprechen den Musen den Sieg zu und die Periden werden in Elstern verwandelt (Ovid, Metamorphosen, Buch 5, 294-678).

7 Vgl. zu dieser Einschätzung auch Meier 1893: 840, ebenso seine Beschreibung der tiefen Verwurzelung des agonistischen Prinzips in der griechischen Gesellschaft. 
kampfes verbergen. Jeder Grieche gehörte verschiedenen sozialen Gruppen an. Er war eingebunden in den Genos, seine Sippe, die Phyle, seinen Stamm, und schließlich seine Polis - ein Begriff, der sich mit Stadt nur unzureichend übersetzen lässt, denn wie auch Genos und Phyle definierte sich die Polis nicht durch topographische Grenzen oder einen politischen Einflussbereich, sondern allein als Personalverband (Meyer 1980: 68-70). ${ }^{8}$ Das gleiche gilt für das Griechische, das keinen Staat, kein Territorium, keinen politischen oder ökonomischen Einflussbereich und keine Ethnie bezeichnet, sondern einen Verband von Menschen, die sich zu einem bestimmten Kult und einer bestimmten Kultur bekannten. Während es Territorialstaaten zur Selbstdefinition ausreicht, die eigenen Grenzen durch militärische Mittel zu erkämpfen, und Monarchien sich durch Anerkennung und Durchsetzung der Gewalt des Herrschers selbst bestimmen, brauchen Systeme oder Gesellschaften, die sich als Personalverbände verstehen, die Einigung auf Codes, die von allen Beteiligten möglichst gleich und eindeutig verstanden werden: auf einen gemeinsamen Götterhimmel z.B., auf gemeinsame Gesetze oder eine gemeinsame Sprache.

Die musikalische Agonistik begann in dem historischen Augenblick im 7. Jahrhundert v. Chr. aufzublühen, als sich die griechische Kultur, die ohnehin in einer zerklüfteten Landschaft und auf Hunderten von Inseln früh schon das Problem der Kommunikation kannte, weiter ausdehnte und Kolonien bildete, rund um das Mittelmeer und das Schwarze Meer, von Marseille bis Zypern, von der Krim bis nach Ägypten. Die Verbreitung einer Kultur über große räumliche und damit auch zeitliche Distanzen und die Nachbarschaft zu sehr unterschiedlichen, lokal vorgefundenen Kulturen gefährdet das Verstehen, die Eindeutigkeit der Codes. Die panhellenischen Spiele hatten für die griechische Kultur eine überlebenswichtige Funktion: Sie produzierten angesichts des Problems der Verbreitung Verstehen durch das Herstellen von Konsens. Auf den Treffen der Agonisten wurden immer wieder neu Grenzen gezogen zwischen dem, was als Griechisch, und dem, was als Nicht-Griechisch gelten konnte.

Die Sieger der Agone, d.h. diejenigen Kulturschaffenden im wahrsten Wortsinne, die bei den Wettkämpfen am eindeutigsten verstanden wurden, die die meisten gleichen Anschlusshandlungen auf sich vereinigen konnten, zogen mit der so gewonnenen Autorität und göttlich begründeten Definitionsmacht durch das Land und stimmten die Kultur auf sich ein. Sie sorgten für ein eindeutiges Verstehen kultureller Codes und verbreiteten diese Kon-

8 Auch wenn in Athen später die Phylen aufgelöst und als Stadtteile neu organisiert wurden, blieb das Prinzip, dass die Menschen nicht nur Familie und Stamm, sondern auch den Staat (aus-)machten. 
sens-Kunst so lange, bis das Erfolgsmedium Macht, der Ruhm, den ein Sieg in Sparta, Delphi oder Athen bedeutete, verblasste. Die Sieger vieler wichtiger Agone wurden Teil der Tradition, ihre Namen sind bis heute überliefert. Bezeichnend ist, dass die Spielweisen, die entwickelt wurden, Nomoi, übersetzt: Gesetze genannt wurden und Terpander, der Sieger vieler Wettbewerbe, als Nomothetes, als Gesetzgeber angesehen wurde (Ps.-Plutarch, Peri mousices, 1132C ff., bes. 1133B; vgl. auch Platon, Politeia, 424c ff.; West 1992: 337). Reisende Techniten brachten ihre Kunst bis an die Grenzen der bekannten Welt. So berichtet Plutarch (Alexander, Kap. 72), dass bei einem Aufenthalt Alexanders des Großen in Ekbatana (das heutige Hamadan im westlichen Iran) 3000 Techniten zu Festspielen zusammen kamen - eine gewaltige Zahl angesichts der relativ kleinen Trägerschicht der Kultur und der Entfernung vom eigentlichen Kern der Kultur. Als die griechische Kultur in der römischen aufging, erreichte die Agonistik ihre größte Verbreitung. Aus dem 3. Jahrhundert n. Chr. stammt das Grab eines 16-jährigen Choraules in Köln, eines Aulos-Spielers, der Chorgesang begleitete. Der Knabe kam aus dem kleinasiatischen Mylasa und war auf seiner Tournee zusammen mit seinem Vater bis an die Grenzen Germaniens gekommen (Herz 1990: 181/187).

Mit der Eroberung der griechischen Siedlungsgebiete zwischen Süditalien und Kleinasien durch das römische Reich endeten die griechischen Agone nicht. Der römische Staat definierte sich als territoriale Macht, er konnte daher eklektizistisch die verschiedenen Kulturen der eroberten Gebiete in sich aufnehmen. Das Griechische blieb als die wichtigste Kultur des römischen Reichs bestehen. Die Agone, die auf römischem Territorium und auch für Römer veranstaltet wurden, waren letztendlich griechische Spiele. Die Römer übernahmen lediglich das Protektorat über die Technitenverbände und garantierten ihre Privilegien - sicherlich auch, um sie zu kontrollieren. In Rom selbst organisierte sich eine »Societas cantorum Graecorum« (Wille 1967: 362). Doch erst mit der römischen Kaiserzeit blühten die Wettbewerbe mit einer großen Zahl von Neugründungen stark auf und es entwickelten sich Ansätze einer genuin römischen Agonistik. Die große Zahl der neu gegründeten Agone diente der Verehrung des zum Gott erhobenen Kaisers. In einem Reich, dessen Grenzen immer schwerer zu kontrollieren waren, dessen Sprachen, Kulturen, Gottheiten unüberschaubar wurden, wurde eine, den Gottkaiser ehrende und von ihm legitimierte »Leitkultur « wichtig. Spezielle Preise für lateinische Dichtung sollten die Gleichwertigkeit der römischen Kultur mit der griechischen belegen - ohne jedoch großen oder auch nur nachvollziehbaren Einfluss auf die lateinische Literatur zu erlangen (White 1998: 85). 
Rom, das Zentrum der Macht, erlebte die ersten periodisch wiederkehrenden Wettkämpfe erst unter Kaiser Nero ab $60 \mathrm{n}$. Chr. ${ }^{9}$ Die so genannten Neronien, die in Abständen von fünf Jahren stattfanden und musische, gymnische und hippische Wettkämpfe umfassten, endeten mit dem Tod des Kaisers. Zu sehr waren sie mit der Person ihres Ausrichters, Teilnehmers und wen wundert es - ständigen Siegers verknüpft (Wille 1967: 340-350). Erst Domitian richtete $86 \mathrm{n}$. Chr. mit den alle vier Jahre statt findenden ludi Capitolini den ersten dauerhaften und wichtigsten römischen Agon nach griechischem Muster ein, der in der Rangfolge gleich nach den vier klassischen panhellenischen Spielen gezählt wurde (Leppin 1992: 171; umfassend dazu Caldelli 1993). Spätere Kaiser gründeten - als Teil ihres Herrscherkultes - weitere Agone (Herz 1990: 184f.), so dass Rom im 2. und 3. Jahrhundert n. Chr. zur wichtigsten Wettkampfstätte im Westen wurde (Leppin 1992: 171). Die griechische Agonistik endete zusammen mit dem römischen Gottkaisertum. Das aufkommende Christentum stand dem heidnischen Kult der Spiele kritisch gegenüber (Herz 1990: 185). Ihre Funktion für die Produktion eines den Staat vereinigenden kulturellen Konsens hätte das Christentum, das 391 von Kaiser Theodosius als Staatsreligion eingeführt wurde, in West- wie auch in Ostrom übernehmen können - wäre nicht die Stadt Rom kurz darauf von den Westgoten überrannt worden.

Die Bedeutung der Musenkünste für die Selbstdefinition ihrer Gesellschaft war den Griechen selbst bewusst. Im letzten Viertel des 5. Jahrhunderts v. Chr. entstand ein Streit zwischen Tradition und Innovation, der nicht zuletzt durch die Entwicklung der Wettkämpfe ausgelöst wurde. Die Demokratisierung hatte eine Verbreiterung der kulturellen Trägerschicht mit sich gebracht, die nach einfacheren Codes verlangte, um Verstehen zu sichern. Als erster warnte Damon vor den gesellschaftlichen Folgen, die Änderungen in der Musik nach sich zögen, und Platon spricht, sich auf Damon berufend und durch den Mund des Sokrates, den Gründungssatz der Musiksoziologie, dass man Neuerungen in der Musik scheuen solle, denn noch nie seien die Gesetze der Musik geändert worden, ohne dass sich auch die Gesetze der sozialen Ordnung geändert hätten (Platon, Politeia, 424c). Aus diesem Grund fordert er auch in seiner Schrift Nomoi, dass bei Wettkämpfen nicht der Geschmack der Mehrheit entscheiden solle, wie dies z.B. bei Wettbewerben in griechischen Siedlungsgebieten in Sizilien und Italien der Fall sei. Die Masse falle zu leicht auf denjenigen herein, der ihr Genuss

9 Nichtperiodische Agone zu besonderen Anlässen veranstalteten in Rom allerdings bereits Caesar und Augustus (Meier 1893: 866). Augustus richtete mit den Actia einen alle vier Jahre in Nikopolis stattfindenden Agon zum Gedenken an seinen Sieg in Actium ein. 
durch seine Kunststückchen bereitet. Vielmehr sollten die Gebildetsten, Tugendhaftesten und Tapfersten als Richter den Sieger bestimmen. Musik habe einen pädagogischen, charakterbildenden Effekt, der durch den Massengeschmack viel zu leicht korrumpiert werde (Platon, Nomoi II, 657d-659d). Platon versuchte vergeblich, die Eindeutigkeit von Codes, die Profanisierung ihrer göttlichen Herkunft durch handwerkliche Kunststückchen, durch Einschränkung von Verbreitung mit Hilfe von Expertenjurys zu bewahren. Eine Generation später, in Aristoteles' Politika, ist von der Musik bei Wettbewerben nur noch als Genussmittel die Rede, als bloßes Präsentieren von Kunstfertigkeit professioneller Musiker, das des Freien Bürgers nicht würdig sei, denn es sei gemein und mache gemein (Aristoteles, Politika VIII, 1341b 10-15).

Zum ersten Mal in der Geschichte begegnet uns hier eine Diskussion der Differenz von Qualität und Quantität, aus der heraus sich die Dynamik von Wettbewerben speist. Es soll der Beste gewinnen. Doch die Beurteilung von Qualität - ob man sie ethisch definiert, wie Platon und Aristoteles, oder handwerklich oder künstlerisch-ästhetisch - fragt nach einem komplexen Code, der fehlerfrei beherrscht, d.h. eindeutig verstanden wird. Gleichzeitig kann der Gewinn nur durch einen Konsens erzielt werden, der wiederum durch einen auf das Allgemeinverständliche reduzierten und daher wenig eindeutigen Code gefördert wird. In dem Problem, das rechte Maß von Qualität und Quantität, von Verstehen und Verbreitung zu finden, liegt die gesellschaftliche Funktion musischer Wettbewerbe begründet. Weder eine reine Expertenkultur, die einen beschränkten Code als traditionell betoniert und ihn in immer größerer Komplexität bei immer größerer Eindeutigkeit des Verstehens, aber um den Preis des Rückgangs von Verbreitung durchführt, noch die Massenkultur, die immer neue, einfache, vieldeutige Codes in sich aufsaugt und zu einer Beschleunigung der Innovation von Äußerlichkeiten und damit zur Auflösung der Tradition durch Beliebigkeit führt, ist für ein soziales System und letztendlich für eine Gesellschaft wünschenswert.

\section{Hermetik: Vom Pui zum Meistersang und zurück}

Überschaubare, klar hierarchisch gegliederte Systeme wie z.B. die Höfe des Adels im Mittelalter brauchen an sich keine organisierten musikalischen Wettbewerbe. Sie produzieren aus sich selbst heraus genug Konkurrenz und sind hermetisch genug, um eine Stabilität des Systems zu gewährleisten. Höfische Musik und Dichtkunst suchen nicht die Verbreitung, sondern eher 
das Gegenteil: die Privatheit. Durch musikalische Fähigkeiten versucht der Höfling, die Aufmerksamkeit des Ranghöheren, der Hohen Dame und durch sie des Lehnsherrn zu gewinnen. Er beweist seine Liebe und Treue, um das Vertrauen der Besungenen zu erhalten. Was uns in mittelalterlicher Epik und Lyrik als Liebesdienst verbrämt begegnet, war in der Realität nichts anderes als eine durch das zivilisatorische Ritual der Liebe gebändigte Machtpolitik. ${ }^{10}$ Musik diente der individuellen Distinktion, der eigenen Abgrenzung und der Ausgrenzung der Mitbewerber. Sie war umso erfolgreicher, je mehr sie die übrigen Zuhörer vom Konsens zwischen Sänger und Besungener ausschloss. Das Geheimnis, das die Sänger um den Namen ihrer Geliebten machten, zeigt, dass die Verbreitung von Bedeutungen sogar ausgeschlossen werden sollte. Der singende Höfling musste Konsens nur mit einem oder einer herstellen, musste nur dem Herrn oder der Herrin gefallen.

In Dichtungen, die für Kontexte außerhalb der Liebeshöfe geschrieben wurden, erscheinen allerdings prompt Merkmale der Konkurrenz: politische und moralische Fragen werden von Troubadours und Trouvères meist in Streitgedichten wie den Sirventés und den Tenzonen bzw. den jeux partis ausgetragen. Die verwendeten Codes sind - abgesehen vielleicht vom intellektuelleren jeu parti - entsprechend einfach: Gern werden Konkurrenten oder politische Gegner mit den übelsten Schimpfworten überhäuft. ${ }^{11}$ Auch diese Konkurrenzen haben die Funktion, Gemeinsamkeit durch den Ausschluss anderer herzustellen, wie Catherine Leglu (1996: 64) feststellt.

Tatsächliche Wettbewerbe entstanden, als Verbreitung und Verstehen aus dem Gleichgewicht zu geraten drohten. Der wachsende Wohlstand des Bürgertums ermöglichte Muße und ließ ein Standesbewusstsein wachsen, das auf eine Aneignung der höfischen Kultur drängte. Die Entstehung der Puis, der städtischen Sängerwettstreite, die seit der ersten Hälfte des 13. Jahrhunderts in Nordfrankreich nachgewiesen sind, ist ein typisches Übergangsphänomen. Die Übernahme von Codes von einem anderen sozialen System ist immer mit Verstehensproblemen verbunden. Die Welt des Dienstes an Gott, dem Feudalherrn und der Hohen Frau, in der der Adel lebte, entsprach nicht der des Bürgertums. Die Herkunft der Lieder aus dem Adel und das damit verbundene Prestige sorgten zwar für ihren Erfolg, bewirkte jedoch auch Verstehensprobleme. In der Stadt konnte höfische Liebesdichtung die Funktion des Dienens, der Monopolisierung von Verstehen zwischen Dich-

10 Eine aufschlussreiche, wenn auch späte Quelle zur Funktion höfischer Kultur ist Baldesar Castigliones Cortegiano vom Anfang des 16. Jahrhunderts (Castiglione 1955: besonders Buch IV, Kap. 4f.).

11 Das Beschimpfen von Konkurrenten in Gedichten und Liedern hat eine Tradition, die mit dem »Dissen« der Rapper bis in die Gegenwart reicht. 
ter und Rezipient, nicht mehr erfüllen. Der neue soziale Kontext brauchte ein anderes Verstehen, das Verbreitung ermöglichte und dennoch so einschränkte, dass Distinktion möglich blieb. Diese Funktion erfüllten die Puis.

Ein Zentrum dieser Entwicklung war die nordfranzösische Stadt Arras, die sich im 12. Jahrhundert zu einem wichtigen Handels- und Bankenzentrum entwickelt hatte. Eine große Anzahl erhaltener Texte lassen sich dem Pui von Arras zuschreiben. Aus den Textquellen wissen wir einiges über die Sänger der Puis, die zu einem großen Teil aus dem Bürgertum kamen, also Laien waren, zu denen jedoch auch einige Adelige und fahrende Sänger gehörten. Ein erstes Statut, das Aufschlüsse über den Ablauf der Wettbewerbe gibt, ist allerdings erst vom Londoner Pui um 1300 erhalten (vgl. Rösler 1921). Der Londoner Pui war sicherlich keine Veranstaltung, die für Berufsmusiker attraktiv war, denn die Teilnehmer mussten verschiedene Gebühren entrichten und es gab - soweit bekannt - außer der Ehre der Krönung des Liedes keinen Preis zu gewinnen. Zudem war der Londoner Pui außerordentlich hermetisch organisiert. Nichtmitglieder durften zwar noch am vorausgehenden Festschmaus teilnehmen, mussten zum Wettsingen jedoch den Saal verlassen. Hier imitierte der Pui in gewisser Weise die Abgeschlossenheit der höfischen Gesellschaft. Frauen allerdings waren nicht zugelassen und das, obwohl der Pui laut Statuten nicht nur zu Ehren Gottes, der Jungfrau Maria, aller Heiligen, des Königs und Adels sowie der Stadt London abgehalten wurde, sondern auch »por loial amour ensaucier «: um die treue Liebe zu verbreiten (Rösler 1921: 115). Schließlich ging es anders als am Hofe - um das Prinzip und nicht um tatsächliche Werbung. Jedes Jahr wurde ein angesehener Bürger als prince gewählt, der den Vorsitz führte. Ihm zur Seiten standen in London bis zu 15 Juroren, von denen - so eine spätere Version des Statuts - mindestens zwei oder drei in der Musik erfahren sein sollten. Der Sieger wurde zu Pferd durch die Stadt geführt, die erfolgreiche, "gekrönte« Chanson, die chanson couronnée, als Vorbild an der Wand des Festsaals aufgehängt (vgl. van der Werf/Frobenius 1983; Rösler 1921: 114).

Während die hermetische Organisation der Puis die Verbreitung begrenzte und damit auch den Erfolg, das Prestige des Genres sicherte, mussten neue Codes, ein neuer Konsens des Verstehens herausgebildet werden. In der Stadt wurde eine neue soziale Funktion der Musik gegründet. Das Werben um die Gunst des Höhergestellten machte in einer Gesellschaft von de jure weitgehend Gleichen kaum Sinn und das Werben um die Gunst der Bürgerstochter hätte die Musik erniedrigt. Stattdessen wird sie zu einer Kunst: es geht jetzt vor allem um die Faktur des Liedes, seine Form und Regelhaftigkeit - die immer mehr kodifiziert wird. Das kritisierte im Über- 
gang von höfischer zu städtischer Kultur z.B. der Dichter Robert de le Piere aus Arras (gest. 1258) in einem Gedicht:

»Jene, die mich für mein gekröntes Lied tadelten, haben nur nicht genau geprüft, was ich fühle und bis an mein Ende denken werde. Freilich singe ich auch nicht für sie. Für Euch, Schöne, ist [mein Lied] erfunden worden - und Ihr habt es nicht getadelt! Darauf bin ich stolz« (zit. n. Räkel 1997: Sp. 1911).

Ähnliche Formen von Wettkämpfen sind mit den jeux floraux in Toulouse und den Rederijkers in den Niederlanden bekannt. Auf die Existenz von vergleichbaren Wettbewerben im deutschsprachigen Raum kann man nur aus der Literatur wie der anonym überlieferten Geschichte vom Sängerkrieg auf der Wartburg schließen. Es ist jedoch nicht auszuschließen, dass der Wartburgkrieg eine literarische Fiktion des späten 13. und 14. Jahrhunderts ist (vgl. z.B. Müller 1996: 39). Mit Sicherheit fiktiv ist der ebenfalls in einem höfischen Kontext angesiedelte Sängerwettstreit, den Jehan de Le Mote in seinem Parfait du Paon (1340) schildert (Räkel 1997: Sp. 1915). Gesichert ist dagegen der Wettbewerb am 1400 gegründeten Liebeshof Karls VI. von Frankreich (Green 1983: 93-97). Offenbar wirkten die bürgerlichen Puis auf die höfische Kultur zurück, machten dort die implizite Konkurrenz explizit, ritualisierten sie in einem Prozess der Zivilisation im Sinne von Norbert Elias.

Spätestens seit dem Beginn des 15. Jahrhunderts gibt es vor allem aus süddeutschen Städten Hinweise auf Vereinigungen von Meistersängern. Ihre - selbst überlieferte - Entstehungslegende will, dass Heinrich von Meißen, genannt Frauenlob, in Mainz die erste Singschule abhielt. Diese Legende wird jedoch erst etwa 200 Jahre nach Frauenlobs Tod 1318 in einer Quelle greifbar (Hahn 1985: 19). Konkrete Fakten über städtische Meistersängervereinigungen liegen erst ab etwa 1500 vor. Das betrifft auch die Kenntnis von den Sängerwettstreiten der Meistersinger. Der Nürnberger Dichter Hans Folz vermerkt unter einem seiner Lieder, es sei 1496 in einer Singschule um den Preis eines Kleinods vorgetragen worden (ebd.: 27/30). Auch Schulordnungen, die bezeugen können, dass und wie die Sängerwettbewerbe der Meistersänger abgehalten wurden, sind erst ab dem 16. Jahrhundert überliefert.

Die Singschulen der Meistersänger stehen - wie die früheren Pui - an historischen Stellen des Übergangs von Codes, reduzieren Verbreitung und sichern Verstehen. Sie markieren die Übernahme der Kunst der fahrenden Sangspruchdichter durch bürgerliche Laien. Ihre Blüte erlebten sie jedoch in einer anderen Zeit des Wandels, die ein gänzlich neues Verstehen forderte, 
und die bald nach 1500 einsetzte: die Reformation. Die Singschulen hatten nur eine kleine Jury mit drei, maximal vier so genannten Merkern, die ausgesprochene Experten waren und noch dazu auf schriftlich niedergelegte Regelwerke, die Tabulaturen, zurückgriffen. Sie verglichen die formale Richtigkeit des vorgetragenen Tons, d.h. der Sangweise, aber auch die inhaltliche Richtigkeit. Jede Abweichung von tradierten Formen und Inhalten wurde mit Strafpunkten belegt. Die Hauptsingen, die in Kirchen nach dem sonntäglichen Gottesdienst abgehalten wurden und in denen nur Lieder mit Inhalten aus der Bibel vorgetragen werden durften, dienten auch zur Kontrolle religiöser Bildung. Ein Merker war ausschließlich dazu da, die Übereinstimmung der vorgetragenen Verse mit der Lutherbibel zu vergleichen. Als letzte wurde erst 1875 die Memminger Meistersingergesellschaft aufgelöst der letzte Meistersinger, Friedrich Hummel, starb 1922. Wie bei allen anderen Gesellschaften hatte sich der Schwerpunkt ihrer Aktivitäten nach dem Ende der religiösen Wirren des 17. Jahrhunderts von den Singschulen auf das Theaterspiel verlagert (Hahn 1985: 86).

Auch die Gegenreformation förderte die Bildung von Wettbewerben, so z.B. den um 1575 eingerichteten Puy de Ste Cécile in Evreux. Sänger der Kathedrale und Einwohner der Stadt gründeten 1570, kurz nach dem Ende des auch für die Zukunft der Kirchenmusik entscheidenden Tridentinischen Konzils 1563, mit Zustimmung des Kapitels eine Bruderschaft, die es sich zur Aufgabe machte, den Gottesdienst musikalisch zu unterstützen. Der von dieser Bruderschaft ausgeschriebene Wettbewerb belohnte die besten Motetten, Chansons, Airs und Sonette, also mehrstimmige Kompositionen. Die verdienten Stücke wurden in einem Buch gesammelt, damit man sich ihrer erinnere (Fédorov 1954: Sp. 1638-1641). Der Puy von Evreux - zu dessen Preisträgern auch Orlando di Lasso (1575 und 1583) gehörte - hatte nichts mehr mit den mittelalterlichen Veranstaltungen gleichen Namens zu tun. Er existierte bis 1790 und markiert den Übergang von den Sänger- und Dichterwettstreiten des Mittelalters zu den modernen Kompositionswettbewerben. Der Puy de Ste Cécile in Evreux war nicht der erste, aber lange der bedeutendste Wettstreit seiner Art; an Ste Cécile in Rouen (vor 1565) und Ste Cécile in Paris (vor 1575) waren kurz zuvor ähnliche Wettbewerbe gegründet worden. Diese Tradition der Wettbewerbe von Cäcilien-Bruderschaften wurde 1633 mit einem Wettbewerb in Le Mans und 1669 in Caen fortgesetzt. Die heilige Cäcilie war bereits lange vor dem Caecilianismus des 19. Jahrhunderts ein Symbol für die Suche nach einem Konsens über die ideale Kirchenmusik. 


\section{Anpassung: \\ Vom 19. Jahrhundert bis zur Gegenwart}

Seit dem Anfang des 19. Jahrhunderts lässt sich ein sprunghaftes Anwachsen der Anzahl öffentlicher, regelmäßig stattfindender Wettbewerbe feststellen, eine Entwicklung, die nach wie vor anhält. Das Musikinformationszentrum des Deutschen Musikrats (www.miz.org) verzeichnete im Frühjahr 2005 unter der Kategorie »Förderung « allein für Deutschland 280 Wettbewerbe sowie 192 Preise. ${ }^{12}$ Auffällig bei den neu gegründeten Wettbewerben sind die Motivationen, die sich deutlich von Wettbewerben aus der Zeit vor 1800 unterscheiden. Es geht jetzt in der Regel nicht mehr darum, einen Konsens darüber zu erzielen, wer unter hervorragenden, etablierten Musikern der Beste sei. Hauptbeweggründe für Stifter sind seit dem 19. Jahrhundert zum einen die Ausbildung bzw. die Förderung des Nachwuchses sowie zum anderen die Förderung des Laienmusizierens.

Die Tradition der Auslobung von Wettbewerben für junge Musiker begann zunächst auf dem Gebiet der Komposition. Der 1803 von Napoleon aus einem älteren Wettbewerb für bildende Künstler heraus gegründete Prix de Rome, der ursprünglich nur für junge Franzosen unter 30 Jahren zugelassen war, ist sicherlich der älteste noch existierende Preis für Komponisten wenn sich auch seine Modalitäten seit 1971 stark verändert haben. Im Verlauf des 19. Jahrhunderts mehrten sich die Preise und Stipendien zur Unterstützung talentierter Musikstudierender. In Deutschland wurden z.B. die Mozartstiftung (1838), die Mendelssohn-Stiftung (1861) oder die Franz LisztStiftung (1887) gegründet. Die Zeit der großen, regelmäßigen Interpretationswettbewerbe für Solisten und Kammermusikensembles begann 1890 mit dem Rubinstein-Wettbewerb in St. Petersburg.

Die neuen Wettbewerbe zur Nachwuchsförderung sind ein Spiegelbild der Veränderungen des Musikverständnisses und der sozialen Kontexte des Musizierens. Ein Faktor ihres Entstehens und ihrer sozialen Notwendigkeit war sicherlich das mit der Aufklärung aufgekommene neue Kunstverständnis. Originalität oder gar Genialität lassen sich zwar konstatieren, aber kaum messen, geschweige denn vergleichen. Das Ideal vom Kunstwerk als einem individuellen, originellen Werk birgt ein Problem des Verstehens. Wenn es darauf ankommt, anders zu sein, und diese Verschiedenheit zu dem Faktor wird, der die Qualität der musikalischen Kommunikation auszeichnet, entstehen hoch spezialisierte, individualisierte Codes, die die Verbreitung

12 Die Landeswettbewerbe von »Jugend musiziert « sind als einzelne Wettbewerbe aufgeführt. 
gefährden. Eine mögliche Lösung dieses Problems der drohenden Destabilisierung der Kommunikation ist die Einführung einer Übergangszeit zwischen Ausbildung und Meisterschaft, in der mit Hilfe von Wettbewerben ein Vorwand geschaffen wird, Originalität zu bewerten und damit zu kontrollieren. Wettbewerbe werden so zum »Erziehungsprinzip« (Rohlfs 1998: Sp. 1996). In einem sozialen System, das nach wie vor einen romantischen Kunstwerkbegriff zelebriert, sorgen Wettbewerbe für die notwendige Bodenhaftung, für den Konsens, wie originell Kunst sein darf und wie groß die zumutbaren Verstehensprobleme sein dürfen. Da diese Wettbewerbe in der Regel mit Expertenjurys besetzt sind, definieren sie jedoch auch die Grenzen nach unten, verhindern allzu einfache Codes, eine allzu mühelose Verbreitung und erhalten damit den Expertenstatus von Kunstmusik. Joachim Kaiser beschreibt die Funktion moderner Wettbewerbe in einer Diktion, die bezeichnender Weise an den Sozialdarwinismus vergangener Zeiten erinnert:

»Wer nicht fähig ist, sich herauszuheben, wer nicht genügend Individualität besitzt, einen eigenen Interpretationston zu finden, wer den technischen Anforderungen nicht genügt, der empfängt in den Wettbewerben eine schmerzliche, aber heilsame Lehre. [...] Wettbewerbe lehren doch, wer gesunde Kraft, auffallende Individualität und wohlerworbenes Können in die Waagschale zu legen hat« (Kaiser 1979).

Ist ein Musiker jedoch erst einmal mit genug Erfolg ausgestattet, wird er sich hüten, an direkten Vergleichen teilzunehmen. Er hat dann genug eigene Definitionsmacht gesammelt, ist vom Gesellen zum Meister aufgestiegen. Er ist im Idealfall unvergleichlich geworden und zum Maßstab, an dem der Nachwuchs gemessen wird.

Zum Anstieg der Zahl der Wettbewerbe dürfte jedoch auch die geänderte berufliche Situation der Musiker beigetragen haben. Mit der Verbürgerlichung der Musikpflege im 19. Jahrhundert änderte sich das kommunikative Umfeld der Musikpraxis. Im Gegensatz zum Spiel in der Kammer oder im Salon eines Fürsten bietet das öffentliche Konzert vor einer möglichst groBen Masse zahlender Zuschauer nur noch sehr eingeschränkte Möglichkeiten der strukturellen Koppelung. Der Schlussapplaus gibt dem Musiker nur global Auskunft über seine Leistung. Zudem fehlt der egalisierten Hörermasse des Konzerts, deren sozialer Rang, ausgedrückt in der gewählten Sitzplatzkategorie, sich nicht auf eine Autorität in Geschmacksdingen erstreckt, die Wortführerschaft des Fürsten oder Gastgebers einer Akademie oder eines Salons, die zuvor die Kontingenz der Kommunikation zwischen Musikern und Hörern stark einschränkte und damit die Verständigung eindeutiger machte. Diese Funktion mussten im 19. Jahrhundert Musikkritik und Wettbewerbe 
übernehmen. Sie regulieren als gesellschaftlich legitimierte Institutionen der Zuschreibung von Erfolg die Verbreitung von Kunst.

Ein drittes Problem der Kommunikation zwischen Musikern und Hörern, dass seit dem 19. Jahrhundert immer stärker spürbar wird, ist die Zunahme der Zahl der Hörer, zunächst durch das bürgerliche Konzert, dann durch die Massenmedien, und ebenso die Zunahme der Zahl der Musiker durch den Übergang der Ausbildung vom klassischen Lehrer-Schülerverhältnis auf private und staatliche Konservatorien. Was als Nachwuchsförderung durch Wettbewerb schöngeredet wird, ist nicht viel mehr als eine harte Selektion, die verhindert, dass in der Kommunikation aus zu vielen Musikern Künstler werden. Wettbewerbe übernehmen hier eine Funktion, die das System der Kommunikation zwischen Musikern und Hörern aufgrund des Originalitätsund Individualitätsideals selbst nicht leisten kann. Ob Wettbewerbe jemals die Originalität und Individualität eines Musikers tatsächlich gefördert haben, sei dahingestellt. Liest man die Studie Hans Günther Bastians zu »Jugend musiziert«, scheint es darum auch nicht zu gehen (Bastian 1987; vgl. auch Cossé 1981).

Allerdings befinden wir uns heute in der paradoxen Situation, dass die Verbreitung von Wettbewerben selbst so zugenommen hat, dass diese kaum noch wirksam die Verbreitung von Codes kontrollieren können. Abgesehen vom Gewinn einiger weniger international renommierter Preise bedeutet ein Platz auf dem Siegertreppchen kaum noch einen Zugewinn an Erfolg, denn viele andere Musiker haben ähnliche Auszeichnungen vorzuweisen (vgl. Cossé 1981: 6). Die Kunstexperten verlieren an Einfluss; längst haben sich Umsatzzahlen als wichtigster Indikator für Erfolg etabliert, doch diesen Erfolg erhält man in einem Prozess, der zwar auch Wettbewerb genannt wird, in dem jedoch ganz andere Regeln der Kommunikation herrschen.

Neben diesen Wettbewerben für Musiker, die eine professionelle Karriere eingeschlagen haben, wurden seit der Mitte des 19. Jahrhundert zunehmend auch Preise für Laienchöre bzw. -musikensembles ausgeschrieben. Grundlage dieser Entwicklung war die Zunahme musikalischer Fähigkeiten in immer breiteren Bevölkerungsschichten durch die Einführung eines allgemeinen Musikunterrichts in verschiedenen europäischen Ländern. Eine Geschichte der musikalischen Wettbewerbe sollte zumindest die britischen (und später auch US-amerikanischen) Brass Band Contests und die Gesangskonkurse der belgischen, französischen und deutschen Männergesangsvereine erwähnen.

Wird Musik in der Freizeit, ohne existentielle Motivation getrieben, überlagern häufig andere soziale Funktionen das Bemühen um eine ideale 
musikalische Kommunikation. Rückt z.B. die Geselligkeit in den Vordergrund, entsteht eine gewisse Beliebigkeit der musikalischen Codes. Man singt oder musiziert zum Spaß oder zur Erholung und legt damit Wert auf ganz andere Dinge als jemand, der sich den Status des Künstlers erarbeiten will oder erarbeitet hat. Den Laienmusikwettbewerben kann so durchaus auch - aus dem Blickwinkel des Kunstsystems - eine erzieherische Funktion zugesprochen werden, die - durch den Erfolg motiviert - Laien an kunstmusikalische Standards heranführt und so, trotz der Verbreitung der Kommunikation, Verstehen im Sinne des Kunstsystems aufrecht erhält und Beliebigkeit in der Interpretation einschränkt.

Dass die Kategorien des Kunstsystems in der Laienmusik jedoch nur ein Aspekt der Kommunikation sind, zeigt der Streit um die Wettbewerbe der deutschen Männerchöre im 19. Jahrhundert. Philipp Spitta und Otto Elben z.B. sahen am Ende des 19. Jahrhunderts die Chorbewegung im Kontext eines ganz anderen sozialen Systems. Ihnen ging es nicht um Kunst, sondern um Nation und Nationalcharakter. Spitta sah den Impuls der Männerchorbewegung im »Bewußtsein vom Werthe der deutschen Nation«, der »Liebe zum Vaterlande «, dem »Streben, die nationalen Tugenden zu pflegen« (Spitta 1894: 311). Aus dieser nationalen Idee heraus stiftete Kaiser Wilhelm II. 1895 einen Preis für Männerchöre - obwohl Wettbewerbe in der Bewegung selbst heftig umstritten waren und vom Deutschen Sängerbund abgelehnt wurden (Heemann 1992: 99-101). Otto Elben, Gründungsmitglied und erster Vorsitzender des 1861 gegründeten Deutschen Sängerbundes, wurde nicht müde zu betonen, dass dieser Brauch dem Männerchorwesen fremd sei:

»Man kann auch einräumen, daß der Sporn solchen Kampfes ein für das Kunststreben eindringlicher sein kann [gesperrt im Orig., D.H.] - und dennoch wird man nicht umhin können, diesen Wettstreit unter die Schatten des Sängerwesens einzureihen! Die wahre Kunst hat keinen Gewinn zu hoffen: die Ueberreizung in den Schwierigkeiten der Tonwerke, welche absichtlich schwer komponirt werden, das Uebermaß von auf die Wirkung berechneten Feinheiten fördern nicht mehr die wahre Kunst. Und am allerwenigsten stimmen sie zu der Natur des Männergesangs, dem Natürlichen, Kräftigen, Volksmäßigen. Es ist eine fremde, französisch-belgische Pflanze, welche auf deutschen Boden nicht gehört [...]. Es wäre Verschwendung deutscher Kraft, sich in diese weichliche, eindruckhaschende und doch schaale, übermäßige Verfeinerung einzuleben; die Menschenstimme ist kein Instrument! Noch mehr aber widerstrebt der volksmäßige Karakter unseres Männergesangs, seine Stellung im öffentlichen Leben, sein nationaler Gehalt dieser Schule der Eitelkeit« (Elben 1887: 475f.). 
Nationalität kann man feststellen, nicht jedoch vergleichen und steigern. Das soziale System, das Nationalität im 19. Jahrhundert zu definieren versuchte, suchte Merkmale, nicht Steigerungen des Deutschen. Diese Merkmale fand man im Männergesang und seinem Repertoire, nicht im Streben nach künstlerischer Qualität.

Mit einer Beschreibung des britischen Brass Band Movements nähern wir uns dem Beginn einer Geschichte der Wettbewerbe in der populären Musik. Bis heute ist die britische Blasmusikbewegung vor allem eine Arbeiterkultur. Sie begann gegen Ende der ersten Hälfte des 19. Jahrhunderts mit dem Gedanken der Verbesserung der Bildung der Unterschicht durch Verbreitung musikalischer Fähigkeiten und klassischer, populärer Konzertliteratur. Diese erzieherische Funktion wurde dadurch gestärkt, dass das Brass Band Movement von der Mittelschicht unterstützt wurde, in dem Glauben, dass durch Bildung und (besonders Kunst-)Musik Charakter und Moral der Arbeiterklasse verbessert werden könnten. Namen früher Gewinner von Wettbewerben wie die Mossley Temperance Band oder Bramley Temperance Band weisen auf die Förderung der Arbeiterkultur durch die von der Mittelschicht getragene Temperenzlerbewegung hin (Herbert 2000a: 328; 2000b: 32). Zu einer Bewegung wurde das Spielen in Blechblasensembles jedoch erst mit dem Einsetzten eines wahren Booms von Wettbewerben zu Beginn der 1850er Jahre:

»It is clear that it is this feature of the movement - the idea that bands contest against each other to be judged best or worst, according to commonly understood criteria - which has produced almost all its idiomatic characteristics« (Herbert 2000a: 8).

Angesichts der zunehmenden Verbreitung einer gewissen Form musikalischer Kommunikation dienten Wettbewerbe auch hier dazu, die drohende Vieldeutigkeit von Codes einzuschränken und Verstehen zu sichern. Mit den brass band contests entstanden standardisierte Besetzungen, ein eigenes Repertoire, musikalische Standards, ein Gefühl der Zusammengehörigkeit sowie das Bewusstsein, zu einer nationalen Bewegung zu gehören. Schließlich verhinderten sie auch - trotz der teilweise lukrativen Geldpreise eine Professionalisierung der Bands (vgl. Gammon/Gammon 2000 und Hindmarsh 2000). Die Wettbewerbe wirkten so stabilisierend, dass das Brass Band Movement bis nach dem 2. Weltkrieg zwar immer wieder Zusammenschlüsse von Bands auf regionaler und nationaler Ebene bildete, diese jedoch nie einen alleinigen Vertretungsanspruch hatten. Oft entstanden Zusammenschlüsse allein aus praktischer Notwendigkeit für die Organisation von contests (Russell 2000: S. 82f.). Aufschlussreich ist der Vergleich mit den Strukturen, die diejenigen Teile der deutschen Männerchorbewegung 
ausbildeten, die Wettbewerbe ablehnten. Sie bildeten schnell einen streng hierarchisch strukturierten Überbau von Sängerbünden heraus, der Richtlinienkompetenzen beanspruchte (vgl. z.B. Elben 1887). Zusammengehörigkeit, eine Vereinheitlichung der kommunikativen Codes, lässt sich auch durch Satzungen oder gar Gesetze erzwingen - wenn die gesetzgebende Autorität stark genug ist.

Wettbewerbe grenzen Kontingenz ein, die durch Verbreitung entsteht, indem sie bestimmten Codes zu Erfolg verhelfen, ihnen eine gewisse Definitionsmacht verleihen bzw. ihnen den Vorrang vor anderen geben. Nach diesem Prozess der Selektion müssen die selegierten Codes wiederum zurück in den Prozess der Verbreitung gelangen, denn Erfolg gewinnt nur dann an Bedeutung, wenn die erfolgreichen Codes möglichst weit verbreitet werden. Diese anschließende Verbreitung haben wir bei allen hier besprochen Stationen der Geschichte der musikalischen Wettbewerbe gesehen: bei den Wanderungen griechischer Techniten, der Dokumentation siegreicher Lieder durch Aushang und Kodifizierung bei Puis und Singschulen oder überhaupt der Preisverleihung, die Symbole des Erfolgs überträgt, die bei späteren Kommunikationen - symbolisch generalisiert - implizit oder explizit immer wieder erscheinen. Doch mit der Verbreitung - zeitlich oder räumlich verschärft sich immer wieder auch das Problem des Verstehens, womit eine periodische Wiederkehr von Wettbewerben notwendig wird.

Den Prozess der Verbreitung von anerkannten, im Wettbewerb ausgezeichneten Codes, aber auch schon die Aufmerksamkeit, die ein Wettbewerb per se generieren muss, um Erfolg zu produzieren, kann sich ein anderes Kommunikationssystem zu Nutze machen, das auf maximale Verbreitung seiner Kommunikation angewiesen ist: beispielsweise die Wirtschaft. Die brass band contests in Großbritannien waren von Beginn an ein wichtiger Geschäftszweig der Unterhaltungsindustrie - professionell organisiert, unter Einsatz modernster Mittel der Wertschöpfung (Herbert 2000b: 49-53). Ohne den Unternehmergeist einzelner hätten Wettbewerbe von Blaskapellen kaum eine solche Bedeutung gehabt und es ist fraglich, ob es überhaupt ein Brass Band Movement gegeben hätte. Die contests schufen einen Massenmarkt, der die riesigen Tempel viktorianischer Unterhaltung wie z.B. den Crystal Palace füllte, der den Absatz von Fachzeitschriften, Noten und Instrumenten anschob und Synergien bis hin zu Transportunternehmen für Gruppenreisen schuf. 


\section{Produktion: Castingshows}

In der Geschichte der Ausbildung des Kommunikationssystems der populären Musik hat es immer wieder Wettbewerbe gegeben. Einige werden in diesem Buch vorgestellt: Der gescheiterte Versuch der Funktionäre des Dritten Reichs, die Verbreitung bestimmter Codes der Tanzmusik zu unterbinden und einen Konsens über eine »deutsche Tanzmusik « herzustellen und zu verbreiten (vgl. den Beitrag von Fred Ritzel), der erfolgreiche Versuch, in Zeiten europäischer Einigung ein europaweit akzeptiertes Fernsehformat mit dem European Song Contest zu entwickeln (vgl. den Beitrag von Irving Wolther), oder auch der ebenfalls erfolgreiche Versuch, nationale Eigenheiten angesichts einer Globalisierung der Kultur mit Hilfe der brasilianischen TV-Festivals auszubilden (vgl. den Beitrag von Carsten Heinke). In allen dort erwähnten Motivationen von Wettbewerben populärer Musik geht es nicht um kommunikative Probleme des Systems Pop (im Sinne von Helms 2005) bzw. des Systems der Wirtschaft. Das Kommunikationssystem, das durch den Tausch von Waren und Geld existiert, profitiert von musikalischen Wettbewerben. Es koppelt sich an das System des Wettbewerbs an und versucht, den Erfolg einer Kommunikation zwischen Musikern und Hörern auf das eigene System der Kommunikation zwischen Nachfragern und Anbietern von Waren zu übertragen. D.h. es versucht, die im musikalischen Wettbewerb gewonnene Definitionsmacht für "gute « Musik - durch Verdinglichung der erfolgreichen Kommunikation als Ware - in Tauschwert und damit konkret in Umsatzzahlen und finanziellen Gewinn zu verwandeln. Dass diese Koppelung nicht immer gelingt und weder das System Sound, die Kommunikation zwischen Musikern und Hörern (Helms 2003: 202f.), vom System Wirtschaft, der Kommunikation zwischen Käufern und Verkäufern, gezielt gesteuert werden kann, noch umgekehrt, belegt die gegenseitige Geschlossenheit der Systeme. ${ }^{13}$

Die Konkurrenz, die das System der Wirtschaft durch Festlegung von Codes wie z.B. Preisen für Waren stabilisiert und auch hier Verstehen sichert, war bisher oft Profiteur, nie jedoch Motivation organisierter und formalisierter musikalischer Wettbewerbe ${ }^{14}-$ bis zur Erfindung von Fernseh-

13 So bedeuteten Erfolge beim European Song Contest nicht zwangsläufig auch Erfolge bei der Vermarktung eines Songs, vgl. den Beitrag von Irving Wolther in diesem Band.

14 Charts sind das Erfolgsbarometer der Konkurrenz im System der Wirtschaft. Sie sagen nichts aus über den musikalischen Erfolg, d.h. die Qualität in der Kommunikation zwischen Musikern und Hörern. Vgl. hierzu die Diskussion um die 
formaten wie Popstars und Pop Idols. Um die Verbesserung der Kommunikation zwischen Musikern und Hörern geht es in diesen neuen Wettbewerben ganz offensichtlich kaum. Das Verstehen musikalischer Codes kann nicht zur Disposition stehen, wenn nur bekannte Songhits, die bereits stark mit dem Erfolgsmedium (Definitions-)Macht aufgeladen sind, im Halbplaybackverfahren vorgetragen werden (vgl. den Beitrag von Marc Pendzich in diesem Band), ${ }^{15}$ wenn die Fachjury häufig globale Geschmacksurteile statt konkreter, musikalisch begründeter Wertungen abgibt (vgl. die Beiträge von Ralf von Appen und Lothwesen/Müllensiefen/Tiemann/Matterne in diesem Band), wenn sie den Ausgang der Endrunde zwar beeinflussen, aber nicht bestimmen kann. Das Problem, dass diese Wettbewerbe lösen helfen sollen, liegt in der Kommunikation zwischen Anbietern und Konsumenten.

Die Krise der Musikindustrie in den letzten Jahren entspringt Problemen der Verbreitung. Zum einen wirkt sich die inzwischen extreme Diversifikation des Angebots bei gleichzeitiger Globalisierung des Marktes aus: Der Gebrauchswert der Produkte wird hierdurch gefährdet; es wird immer unklarer, welchen Nutzen der Käufer überhaupt vom Kauf hat, da sich die Bedeutungen zunehmend individualisiert haben. Das Repertoire von Codes mit Konsensbedeutungen, das durch die wirtschaftliche Konkurrenz entsteht: der Mainstream wird durch die Diversifikation immer schwerer wahrnehmbar. Zum anderen hat die Einführung eines neuen Verbreitungsmediums, des Internets, die Verbreitung noch weiter vereinfacht (und Globalisierung und Diversifikation weiter gefördert) sowie gleichzeitig den Warencharakter von Musik überhaupt in Frage gestellt - durch die Loskoppelung der Musik von einer dinglichen Form (wie der CD) und durch die Destabilisierung und teilweise völligen Annullierung des Kommunikationscodes "Tauschwert" bzw. »Preis«.

Die Wettbewerbe der Formate Popstars und Pop Idols sind Medien, die Kommunikation zwischen der Musikindustrie und ihren Konsumenten durch die Einschränkung von Verbreitung wieder wahrscheinlich machen. Im Vergleich zu der problematisch gewordenen Kommunikation im herkömmlichen System der Musikwirtschaft mit verschlungenen Vertriebswegen, komplexen Marketingmaßnahmen und selten aussagekräftigen Marktforschungen schlieBen diese Formate Kommunikation zwischen Anbietern und Konsument wieder kurz: das Publikum wird durch das Televoting unmittelbar an der Herstellung des Produkts »Superstar « beteiligt und muss gleichzeitig dafür

Aussagefähigkeit von Charts als Merkmal von Popularität populärer Musik z.B. in Hamm 1982 und Middleton 1990: 6f.

15 So dass der Informationswert zumindest der musikalischen Parameter Komposition, Text und Arrangement gegen Null tendiert. 
bezahlen. Das Verhältnis der Einnahmen aus den verschiedenen Quellen (vgl. von Appen in diesem Band) macht deutlich: Die Ware, die mit diesen Formaten verkauft wird, sind weniger die CDs und in der Hauptsache auch nicht die Werbeminuten, sondern es ist vor allem das Recht an der Produktion eines »Stars«. Bezahlt wird diese Ware mit Telefonanrufen, dem so genannten Televoting. Einfluss auf Produkte hat der Kunde im System der Wirtschaft immer nur durch sein Kaufverhalten, nur dass hier, in genauer Umkehrung der Verhältnisse des alltäglichen Konsumgeschehens, dieser Einfluss spürbar wird und der Kauf unbewusst geschieht.

Die Kandidaten der Castingshows treten nicht als Persönlichkeiten auf, sondern als zu formender Rohstoff. Ziel des Trainings während des Wettbewerbs, das zeigen die Kommentare der Jury, die von Appen (in diesem Band) gesammelt hat, ist nicht die Ausbildung der Musikerpersönlichkeit, sondern die Versicherung, dass der Prozess der Formung der Ware weiter aufrechterhalten wird. In Castingshows treten nur zum Schein Musiker gegeneinander an. In Wirklichkeit wetteifern verschiedene fiktive Allianzen von Produzenten, denen sich der einzelne Televoter zugehörig fühlt, um die Definitionsmacht bei der Formung von Produkten.

Die Zahl der Produkte, der unterschiedlichen Codes, wird mit jedem Ausscheiden eines Kandidaten geringer. Die Verknappung der Waren steigert die Nachfrage. Die Form des Wettbewerbs stellt sicher, dass trotz der anwachsenden Verbreitung, durch die sich Erfolg im System der Wirtschaft definiert, Verstehen durch immer größere Eindeutigkeit gesichert wird. So kann der Markt, trotz und gerade wegen der Verknappung der Waren, weiter wachsen. Die Fachjury dient dazu, den Schein der Ausbildung aufrecht zu erhalten - nicht zuletzt auch durch Kommentare, die den Widerstand der Televoter provozieren, um den Verkauf von Stimmrechten zu stimulieren. Dass die Jury selbst nicht mit abstimmen darf, ist wichtig für das Fortbestehen des Kommunikationssystems: Fachjurys bewirken, wie oben gezeigt, Hermetik und verhindern die für die Wirtschaft wichtige Verbreitung.

Dass die "Superstars « zumindest in Deutschland nach dem Ende der jeweiligen Sendestaffel kaum kommerziellen Erfolg hatten, war vorhersehbar. Ihr Erfolg lag in dem Spaß begründet, den sie dem Publikum beim Wettbewerb der Entscheidungsträger gegeneinander boten. Mit dem Ende der Show endete auch diese Funktion. Das verkaufte Produkt der Shows war ja die Produktion, nicht die fertige Ware. Auf dem Musikmarkt müssen Alexander, Daniel, Elli und Co. wieder ganz von vorn als Ware aufgebaut werden. Bei diesem Übergang kann den »Superstars« nur ihre Bekanntheit helfen, nicht jedoch eine besondere Qualität als Musiker, die mit dem Wettbewerb nicht attestiert wird. Gerade diese musikalische Kompetenz wird ja durch die Öf- 
fentlichkeit der Produktion der Ware in Frage gestellt. Casting gilt in Deutschland für viele Hörer populärer Musik als ein negatives Label, das der Glaubwürdigkeit und Authentizität der Musiker schadet. Bei keiner Band oder keinem Interpreten auf dem Musikmarkt ist die Tatsache des Castings so bekannt wie bei den ehemaligen Kandidaten. Sie sind und bleiben in der Wahrnehmung des Publikums die "Superstars« und keine wirklichen Stars (vgl. Lothwesen/Müllensiefen/Tiemann/Matterne in diesem Band).

Die Televoter, deren Favoriten die Wahlen gewannen, konnten sich für eine kurze Zeit erfolgreich fühlen. Dieses kleine Gefühl der Befriedigung war im Preis inbegriffen. Wirklichen Erfolg über das Ende des Wettbewerbs hinaus hatte jedoch lediglich das Fernsehformat der Wettbewerbsshow mit Televoting. Die Götter, die die Regeln machen, gewinnen immer, ob sie Apollon oder BMG heißen. Wer sich auf ihr Spiel einlässt, muss seine Haut zu Markte tragen, ob bocksbeiniger Marsyas oder Daniel Küblböck.

\section{Literatur}

Bastian, Hans Günther (1987). Jugend musiziert. Der Wettbewerb in der Sicht von Teilnehmern und Verantwortlichen. Mainz u.a.: Schott.

Caldelli, Maria Letizia (1993). L'agon Capitolinus: storia e protagonisti dall'istituzione domizianea al IV secolo. (= Studi publicati dall'istituto italiano per la storia antica 54). Rom: Istituto Italiano per la Storia Antica.

Castiglione, Baldesar (1955). Il Cortegiano con una scelta delle Opere minori di Baldesar Castiglione. Hg. v. Bruno Maier. Turin: Unione Tipogr. Ed. Torinese.

Cossé, Peter (1981). »Die Sieger stehen sich gegenseitig im Wege.«In: Neue Musikzeitung, H. 4, S. $1 / 6$.

Elben, Otto (1887). Der volksthümliche deutsche Männergesang. Geschichte und Stellung im Leben der Nation; der deutsche Sängerbund und seine Glieder. Tübingen: Verlag der H. Laupp'schen Buchhandlung.

Fédorov, Vladimir (1954). »Evereux. «In: Die Musik in Geschichte und Gegenwart. Bd. 3. Hg. v. Friedrich Blume. Kassel: Bärenreiter (1. Aufl.), Sp. 1637-1647.

Finscher, Ludwig (2003). "Josquin des Prez.«In: Die Musik in Geschichte und Gegenwart. Personenteil, Bd. 9. Hg. v. Ludwig Finscher. Kassel, Stuttgart: Bärenreiter/Metzler (2. Aufl.), Sp. 1210-1282.

Gammon, Vic/Gammon Sheila (2000). „The Musical Revolution of the MidNineteenth Century: From >Repeat and Twiddle< to >Precision and Snap « « In: Herbert 2000a: S. 122-154.

Geck, Martin (2000). Bach. Leben und Werk. Reinbek: Rowohlt.

Green, Richard Frith (1983). »The Familia Regis and the Familia Cupidinis. In: English Court Culture in the Late Middle Ages. Hg. v. Vincent J. Scattergood und James W. Sherborne. London: Duckworth, S. 87-108.

Hahn, Reinhard (1985). Meistergesang. Leipzig: VEB Bibliographisches Institut.

Hamm, Charles (1982). "Some Thoughts on the Measurement of Popularity in Music. In: Popular Music Perspectives 1, S. 3-15. [Auch in: Ders. (Hg.) (1995). Putting Popular Music in its Place. Cambridge, S. 116-130.]. 
Heemann, Annegret (1992). Männergesangvereine im 19. und frühen 20. Jahrhundert. Ein Beitrag zur städtischen Musikgeschichte Münsters. Frankfurt/M. u.a.: Peter Lang.

Heinze, Carsten (2005). »Der Kunstwettbewerb Musik im Rahmen der Olympischen Spiele 1936. «In: Archiv für Musikwissenschaft 62, H. 1, S. 32-51.

Helms, Dietrich (2003). »Auf der Suche nach einem neuen Paradigma: Vom System Ton zum System Sound. «In: Pop Sounds. Klangtexturen in der Pop- und Rockmusik. Basics - Stories - Tracks. Hg. v. Thomas Phleps und Ralf von Appen (= texte zur populären musik 1). Bielefeld: transcript, S. 197-228.

Helms, Dietrich (2005). »)We're Only In It For The Money<. Populäre Musik, Jugendlichkeit und das Geld aus systemtheoretischer Perspektive.« In: Kongressbericht Weimar 2004 (im Druck).

Herbert, Trevor (Hg.) (2000a). The British Brass Band. A Musical and Social History. Oxford: Oxford University Press.

Herbert, Trevor (2000b). »Nineteenth-Century Bands: Making a Movement.« In: Herbert 2000a: 10-67.

Herz, Peter (1990). „Die musische Agonistik und der Kunstbetrieb der Kaiserzeit.« In: Theater und Gesellschaft im Imperium Romanum / Théâtre et société dans l'Empire Romain. Hg. v. Jürgen Blänsdorf in Verbindung mit Jean-Marie André und Nicole Fick (= Mainzer Forschungen zu Drama und Theater 4). Tübingen: Francke, S. 175-195.

Hindmarsch, Paul (2000). »Building a Repertoire: Original Compositions for the British Brass Band, 1913-1998. «In: Herbert 2000a: 245-277.

Huizinga, Johan (1956). Homo Ludens. Vom Ursprung der Kultur im Spiel. Reinbek: Rowohlt.

Kaiser, Joachim (1979). „Was beweisen Musikwettbewerbe?« In: Neue Musikzeitung, H. 5, S. 5.

Komlós, Katalin (1989). »Mozart and Clementi: A Piano Competition and its Interpretation. «In: Historical Performance 2, S. 3-9.

Keym, Stephan (2002). »Häßler, Johann Wilhelm. «In: Die Musik in Geschichte und Gegenwart. Personenteil, Bd. 8. Hg. v. Ludwig Finscher. Kassel, Stuttgart: Bärenreiter/Metzler (2. Aufl.), Sp. 844-847.

Kübler, B. (1934). »Technitai«. In: Paulys Realencyclopädie der Classischen Wissenschaft. 2. Reihe, 10. Halbband. Neue Bearbeitung von Georg Wissowa. Stuttgart: Alfred Druckenmüller, Sp. 2473-2558.

Léglu, Catherine (1996). »A Reading of Troubadour Insult Songs: the Communals Cycle. «In: Reading Medieval Studies 22, S. 63-83.

Leppin, Hartmut (1992). Histrionen: Untersuchungen zur sozialen Stellung von Bühnenkünstlern im Westen des Römischen Reiches zur Zeit der Republik und des Principats (= Antiquitas I. Abhandlungen zur alten Geschichte 41). Bonn: Habelt.

Mainwaring, John (1987). G. F. Händel. Nach Johann Matthesons deutscher Ausgabe von 1761. Hg. v. Bernhard Paumgartner. Zürich: Atlantis (2. Aufl.).

Meier, P. J. (1893). »Agones. «In: Paulys Realencyclopädie der Classischen Altertumswissenschaft. 1. Halbband. Neue Bearbeitung von Georg Wissowa. Stuttgart: Alfred Druckenmüller, Sp. 836-867.

Meyer, Ernst (1980). Einführung in die antike Staatskunde. Darmstadt: Wissenschaftliche Buchgesellschaft.

Middleton, Richard (1990). Studying Popular Music. Milton Keynes, Philadelphia: Open University Press.

Müller, Ulrich (1996). »Der Sängerkrieg auf der Wartburg. Text und Musik einer mittelalterlichen Quelle zu Wagners Tannhäuser.«In: Individuum versus Institution. 
Zur Urfassung (1845) von Richard Wagners »Tannhäuser«. Hg. v. Udo Bermbach, Ulrich Müller und Matthias Theodor Vogt. Leipzig: Leipziger Universitätsverlag, S. 33-46.

Ovidius Naso, Publius (1988). Metamorphosen. Hg. u. übers. v. Hermann Breitenbach. Stuttgart: Reclam.

Pont, Graham (1991). „Handel versus Domenico Scarlatti: Music of an Historic Encounter.« In: Göttinger Händel-Beiträge 4, S. 232-247.

Räkel, Hans-Herbert (1997). »Pui.«In: Die Musik in Geschichte und Gegenwart. Sachteil, Bd. 7. Hg. v. Ludwig Finscher. Kassel, Stuttgart: Bärenreiter/Metzler (2. Aufl.), Sp. 1910-1916.

Rohlfs, Eckart (1998). „Wettbewerbe und Preise. «In: Die Musik in Geschichte und Gegenwart. Sachteil, Bd. 9. Hg. v. Ludwig Finscher. Kassel, Stuttgart: Bärenreiter/Metzler (2. Aufl.), Sp. 1984-2001.

Rösler, Margarete (1921). »Der Londoner Pui.«In: Zeitschrift für Romanische Philologie 41, S. 111-116.

Russell, Dave (2000). » What is Wrong with Brass Bands?: Cultural Change and the Band Movement, 1918-c.1964.«In: Herbert 2000a: 68-121.

Siegele, Ulrich (1999). »Bachmann, Sixt.«In: Die Musik in Geschichte und Gegenwart. Personenteil, Bd. 1. Hg. v. Ludwig Finscher. Kassel, Stuttgart: Bärenreiter/Metzler (2. Aufl.), Sp. 1566-1567.

Spitta, Philipp (1894). »Der deutsche Männergesang.«In: Musikgeschichtliche Aufsätze. Hg. v. dems. Berlin: Gebrüder Paetel.

Van der Werf, Hendrik/Frobenius, Wolf (1983). »Cantus coronatus.«In: Handwörterbuch der musikalischen Terminologie. 11. Lieferung. Hg. v. Hans Heinrich Eggebrecht. Stuttgart: Steiner.

West, Martin Litchfield (1992). Ancient Greek Music. Oxford: Clarendon.

Wille, Günther (1967). Musica Romana. Die Bedeutung der Musik im Leben der Römer. Amsterdam: P. Schippers.

\begin{abstract}
The communication theory of musical contests developed in this paper is inspired by Niklas Luhmann's definition of media as evolutionary means to reduce the fundamental improbability of communication and his description of the evolution of communication as a hydraulic process of balancing the three problems of communication: comprehension, circulation and success. This paper suggests that musical contests help to stabilize social systems whenever the circulation of communication becomes so very likely that codes become increasingly ambiguous and understanding unlikely. Contests produce a consensus. They enrich the successful code with power to define meaning and help thus to reduce ambiguity. This hypothesis is applied to various forms of contests in the history of music: the agones of ancient Greece, the puis of troubadour France and England, the schools of German Meistersinger, the talent contests in art music, the contests of German men's choral societies, the contest of the British Brass Band Movement and finally the TV talent shows of the recent past.
\end{abstract}

